\title{
Least Squares Fitting of Digital Polynomial Segments
}

\author{
Joviša Żunić \\ University of Novi Sad, Faculty of Engineering, \\ Trg D. Obradovića 6, 21000 Novi Sad, Yugoslavia \\ Dragan M. Acketa \\ University of Novi Sad, Faculty of Science, Department of Mathematics, \\ Trg D. Obradovića 4, 21000 Novi Sad, Yugoslavia
}

\begin{abstract}
It is proved that digital polynomial segments and their least squares polynomial fits are in oneto-one correspondence. This enables an efficient representation of digital polynomial segments by $n+3$ parameters, under the condition that an upper bound, say $n$, for the degrees of the digitized polynomials is assumed. One of such representations is $\left(x_{1}, m, a_{n}, a_{n-1}, \ldots, a_{0}\right)$, where $x_{1}$ and $m$ are the $x$-coordinate of the left endpoint and the number of digital points, respectively, while $a_{n}$, $a_{n-1}, \ldots, a_{0}$ are the coefficients of the least squares polynomial fit $Y=a_{n} X^{n}+a_{n-1} X^{n-1}+\ldots+a_{0}$, for a given digital polynomial segment.
\end{abstract}

Key words: Image processing, computer vision, digital polynomial segment, least squares fitting, coding.

\section{Introduction}

Consider a polynomial curve $\gamma$, in the Euclidean plane, with the equation $y=a_{n} \cdot x^{n}+a_{n-1}$. $x^{n-1}+\ldots+a_{1} \cdot x+a_{0}$. A digital curve is defined to be the result of subjecting a polynomial curve to a certain digitization process. The polynomial curve $\gamma$ will be digitized by the method with which the first digital points (points with integer coordinates, often referred to as pixels) below a given polynomial curve are taken. Obviously, this is equivalent to translating the curve by -0.5 in the vertical direction and rounding.

Thus the associated set of digital points for the polynomial curve $\gamma$, called a digital polynomial curve, is defined by

$$
P(\gamma)=\left\{\left(i,\left\lfloor a_{n} \cdot i^{n}+a_{n-1} \cdot i^{n-1}+\ldots+a_{1} \cdot i+a_{0}\right\rfloor\right), i \text { is an integer }\right\}
$$

where $\lfloor k\rfloor$ denotes the greatest integer not larger than $k$.

In general, we will be dealing with finite subsets of $P(\gamma)$ or, more precisely, with digital polynomial curve segments that are obtained by digitizing parts of polynomial curves lying between the lines $x=x_{1}$ and $x=x_{2}$, for some numbers $x_{1}$ and $x_{2}$. Without loss of generality, we can assume that $x_{1}$ and $x_{2}$ are integers. If $x_{2}-x_{1}$ is equal to $m-1$, then the digital polynomial curve segment $P_{m}\left(\gamma, x_{1}\right)$ for the considered polynomial curve $\gamma$ is defined as:

$$
P_{m}\left(\gamma, x_{1}\right)=\left\{\left(i,\left\lfloor a_{n} \cdot i^{n}+a_{n-1} \cdot i^{n-1}+\ldots+a_{1} \cdot i+a_{0}\right\rfloor\right), i=x_{1}, x_{1}+1, \ldots, x_{1}+(m-1)=x_{2}\right\} .
$$

Obviously, $m$ is the number of digital points in the digital polynomial curve segment $P_{m}\left(\gamma, x_{1}\right)$.

One of the earliest problems considered in pattern recognition and image vision was the representation and the recognition of sets of digital (lattice) points that result from digitized straight lines or line segments $([2,3,10,13,18])$. An analogous study for the circles has been also carried out $([9,13,17,19])$. Similar problems have been of interest to number theorists and in fact some of results date back to the time of Bernoulli $([4,5,8])$. 
The purpose of this paper is to give an efficient representation for digital polynomial segments of arbitrary length. For such a representation $n+3$ parameters are used, where the degree of polynomial is assumed to be bounded by $n . P_{m n}\left(\gamma, x_{1}\right)$ can be uniquely represented by $\left(x_{1}, m, a_{n}, a_{n-1}, \ldots, a_{0}\right)$, where $x_{1}$ and $m$ are the $x$-coordinate of the left endpoint and the length of digital polynomial segment, respectively, while $a_{0}, a_{1}, \ldots, a_{n}$ are the coefficients of its least squares polynomial fit $Y=a_{n} X^{n}+$ $a_{n-1} X^{n-1}+\ldots+a_{0}$.

The idea to use the least squares line fit for representation of digital lines is proposed by Melter and Rosenfeld ([15]). Melter et al. ([16]) proved that the least squares line fit uniquely determines the digital line on a segment. Thus, any digital line segment can be uniquely coded by four numbers $\left(x_{1}, n, b_{0}, b_{1}\right)$ where $z_{1}$ is its $x$-coordinate of the left endpoint, the integer $n$ is the number of digital points, and $b_{0}$ and $b_{1}$ are the coefficients of the least squares line fit $Y=b_{0}+b_{1} X$ for the given digital line segment. Let us mention that this representation is an alternative to the well-known representation of digital lines suggested by Dorst and Smeulders ([7]) and to the one by adjacent pairs given by Lindenbaum and Koplowitz ([14]). The least squares method was applied in ([21]) on digital parabola segments (understood as the second degree polynomials - not as a conic curve). Three-dimensional surfaces have been studied in [11].

While there exist constant space representations for digital lines and digital parabolas in literature, no such representation is currently known for digital polynomials of degree greater than two. The representation given in this paper is the first one. Let us mention here that if a plane curve is digitized, then the curve equation and the base description can be used for a trivial representation of its digitization. However, this trivial method has several deficiencies. For illustration, we give two of them:

- in real world image processing an equation of digitized curve is usually unknown. For example: given a $2 D$ picture of "digital pyramid", one can assume that its edges are digital line segments, but the equations of the lines containing its original edges still remain unknown;

- there are infinitely many curve segments (even of different kinds), the digitizations of which give the same digital curve segments. Therefore, a unique method for "one-to-one" mapping between digital curve segments and their representations would be useful (note: the trivial method mentioned above does not satisfy this request).

It is proved in Section 3 that digital polynomial segments and their least squares polynomial fits are in "one-to-one" correspondence. Determination of the least squares fitting polynomial for a given set of points is a linear problem and consequently, it is easily solvable. Unfortunately, the determination of the least squares curve fits for some other curves is usually a nonlinear problem and consequently this is a problem of high computational complexity. Let us note that the representation of digital curve segments by their least squares curve fits is suitable because it is naturally to expect that the least squares fit curve "looks like" the original curve.

\section{Preliminaries}

A finite set of points in the plane is called a scatter diagram. The least squares curve fit for the scatter diagram is a curve, which minimizes the total sum of the squares of the vertical distances from the curve to the data points. The method for determining such a curve is well-known from statistics, e.g. Burr ([6]).

If the scatter diagram is given by $\left\{\left(x_{i}, y_{i}\right), i=1,2, \ldots, m\right\}$ and the equation of its least squares polynomial is $Y=a_{n} X^{n}+a_{n-1} X^{n-1}+\ldots+a_{0}$, then the function

$$
F\left(a_{n}, a_{n-1}, \ldots, a_{0}\right)=\sum_{i=1}^{m}\left(a_{n} x_{i}^{n}+a_{n-1} x_{i}^{n-1}+\ldots+a_{0}-y_{i}\right)^{2}
$$


should be minimized. Thus the following $n+1$ equations:

$$
\frac{\partial F}{\partial a_{n}}=0, \quad \frac{\partial F}{\partial a_{n-1}}=0, \quad \cdots, \quad \frac{\partial F}{\partial a_{0}}=0
$$

must be satisfied. This gives the following system of $n+1$ equations with $n+1$ variables:

$$
\begin{array}{r}
a_{n} \cdot \sum_{i=1}^{m} x_{i}^{2 n}+a_{n-1} \cdot \sum_{i=1}^{m} x_{i}^{2 n-1}+\ldots+a_{0} \cdot \sum_{i=1}^{m} x_{i}^{n}=\sum_{i=1}^{m} y_{i} x_{i}^{n} \\
a_{n} \cdot \sum_{i=1}^{m} x_{i}^{2 n-1}+a_{n-1} \cdot \sum_{i=1}^{m} x_{i}^{2 n-2}+\ldots+a_{0} \cdot \sum_{i=1}^{m} x_{i}^{n-1}=\sum_{i=1}^{m} y_{i} x_{i}^{n-1} \\
a_{n} \cdot \sum_{i=1}^{m} x_{i}^{n}+a_{n-1} \cdot \sum_{i=1}^{m} x_{i}^{n-1}+\ldots+a_{0} \cdot \sum_{i=1}^{m} 1=\sum_{i=1}^{m} y_{i} .
\end{array}
$$

If the previous scatter diagram is a digital curve segment, then $x_{i}=x_{i-1}+1$ (for $i=2, \ldots, m$ ) is satisfied. There are $2 n+1$ coefficients in the previous system. They are:

$$
\begin{aligned}
& S_{0}=\sum_{i=1}^{m} 1 \\
& S_{1}=\sum_{i=1}^{m} x_{i}=x_{1}+\left(x_{1}+1\right)+\ldots+\left(x_{1}+m-1\right) \\
& \ldots \cdots+\left(x_{1}+m-1\right)^{n} . \\
& S_{2 n}=\sum_{i=1}^{m} x_{i}^{2 n}=x_{1}^{n}+\left(x_{1}+1\right)^{n}+\ldots+
\end{aligned}
$$

The coefficients $S_{0}, S_{1}, \ldots, S_{2 n}$ can be calculated recursively by using a well-known technique ([12]), and the previous system becomes

$$
\begin{array}{llll}
S_{2 n} & \cdot & a_{n}+S_{2 n-1} \cdot a_{n-1}+\ldots+S_{n} \cdot a_{0}=\sum_{i=1}^{m} y_{i} x_{i}^{n} \\
S_{2 n-1} & \cdot & a_{n}+S_{2 n-2} \cdot a_{n-1}+\ldots+S_{n-1} \cdot a_{0}=\sum_{i=1}^{m} y_{i} x_{i}^{n-1} \\
& \cdots & & \\
S_{n} & \cdot & a_{n}+S_{n-1} \cdot a_{n-1}+\ldots+S_{0} \cdot a_{0}=\sum_{i=1}^{m} y_{i} .
\end{array}
$$

The coefficients $a_{n}, a_{n-1}, \ldots, a_{0}$ of the least squares polynomial fit can be determined by solving the above system. The following theorem shows that the determinant of the system (1) is different from zero for $m>n$. Consequently, the system (1) has the unique solution in these cases. The system (1) has infinitely many solutions for $m \leq n$, because there exist infinitely many polynomials of degree $n$ that pass $m$ points with pairwise different abscissas. For illustration, we give that this determinant is equal to $\frac{1}{2160} m^{3}(m+1)^{2}(m-1)^{2}(m-2)(m+2)$ for $x_{1}=0$ and $n=2$. Therefore, the system has the unique solution for $m>2$.

Theorem 1 The system (1) can be solved uniquely, whenever $m>n$ is satisfied.

Proof. The determinant of the system (1) can be expressed as follows: 


$$
\begin{aligned}
& \left|\begin{array}{llll}
\sum_{i=1}^{m} x_{i}^{2 n} & \sum_{i=1}^{m} x_{i}^{2 n-1} & \ldots & \sum_{i=1}^{m} x_{i}^{n} \\
\sum_{i=1}^{m} x_{i}^{2 n-1} & \sum_{i=1}^{m} x_{i}^{2 n-2} & \ldots & \sum_{i=1}^{m} x_{i}^{n-1} \\
\ldots & \ldots & \ldots & \ldots \\
\sum_{i=1}^{m} x_{i}^{n} & \sum_{i=1}^{m} x_{i}^{n-1} & \ldots & \sum_{i=1}^{m} 1
\end{array}\right|=\left|\left[\begin{array}{llll}
x_{1}^{n} & x_{2}^{n} & \ldots & x_{m}^{n} \\
x_{1}^{n-1} & x_{2}^{n-1} & \ldots & x_{m}^{n-1} \\
\ldots & \ldots & \ldots & \ldots \\
x_{1}^{1} & x_{2}^{1} & \ldots & x_{m}^{1} \\
1 & 1 & \ldots & 1
\end{array}\right] \cdot\left[\begin{array}{ccccc}
x_{1}^{n} & x_{1}^{n-1} & \ldots & x_{1}^{1} & 1 \\
x_{2}^{n} & x_{2}^{n-1} & \ldots & x_{2}^{1} & 1 \\
\ldots & \ldots & \ldots & \ldots & \ldots \\
x_{m}^{n} & x_{m}^{n-1} & \ldots & x_{m}^{1} & 1
\end{array}\right]\right|= \\
& =\sum_{1 \leq i_{1}<i_{2} \ldots<i_{n}<i_{n+1} \leq m}\left|\begin{array}{lllll}
x_{i_{1}}^{n} & x_{i_{2}}^{n} & \ldots & x_{i_{n}}^{n} & x_{i_{n+1}}^{n} \\
x_{i_{1}}^{n-1} & x_{i_{2}}^{n-1} & \ldots & x_{i_{n}}^{n-1} & x_{i_{n+1}}^{n-1} \\
\ldots & \ldots & \ldots & \ldots & \ldots \\
x_{i_{1}}^{1} & x_{i_{2}}^{1} & \ldots & x_{i_{n}}^{1} & x_{i_{n+1}}^{1} \\
1 & 1 & \ldots & 1 & 1
\end{array}\right| \cdot\left|\begin{array}{ccccc}
x_{i_{1}}^{n} & x_{i_{1}}^{n-1} & \ldots & x_{i_{1}}^{1} & 1 \\
x_{i_{2}}^{n} & x_{i_{2}}^{n-1} & \ldots & x_{i_{2}}^{1} & 1 \\
\ldots & \ldots & \ldots & \ldots & \ldots \\
x_{i_{n}}^{n} & x_{i_{n}}^{n-1} & \ldots & x_{i_{n}}^{1} & 1 \\
x_{i_{n+1}}^{n} & x_{i_{n+1}}^{n-1} & \ldots & x_{i_{n+1}}^{1} & 1
\end{array}\right|= \\
& =\sum_{1 \leq i_{1}<i_{2} \ldots<i_{n}<i_{n+1} \leq m}\left|\begin{array}{lllll}
x_{i_{1}}^{n} & x_{i_{2}}^{n} & \ldots & x_{i_{n}}^{n} & x_{i_{n+1}}^{n} \\
x_{i_{1}}^{n-1} & x_{i_{2}}^{n-1} & \ldots & x_{i_{n}}^{n-1} & x_{i_{n+1}}^{n-1} \\
\ldots & \ldots & \ldots & \ldots & \ldots \\
x_{i_{1}}^{1} & x_{i_{2}}^{1} & \ldots & x_{i_{n}}^{1} & x_{i_{n+1}}^{1} \\
1 & 1 & \ldots & 1 & 1
\end{array}\right|^{2}=\sum_{1 \leq i_{1}<\ldots<i_{n+1} \leq m} \prod_{1 \leq j<k \leq n+1}\left(x_{i_{j}}-x_{i_{k}}\right)^{2} .
\end{aligned}
$$

The second equality follows from Binet-Cauchy's theorem ([1]). In this way, the first determinant is represented as the sum of squares of determinants of order $m$. The last inequality is strict because the summands in the last sum are strictly positive. They are squares of Vandermonde determinants and these determinants are non-zero, since the abscissas $x_{1}, \ldots, x_{m}$ are assumed to be all distinct. $\square$

In the rest of the paper, the inequality $m>n$ will be assumed, but not mentioned. If the scatter diagram is taken to be $P_{m}\left(\gamma, x_{1}\right)$ then the solution of the system (1) will be denoted by $a_{n}(\gamma), a_{n-1}(\gamma)$, $\ldots, a_{0}(\gamma)$.

\section{Least squares representation for digital polynomial segments}

A key question considered in this paper, is whether there exist two different digital polynomial segments, $P_{m}\left(\gamma, x_{1}\right)$ and $P_{m}\left(\beta, x_{1}\right)$, that result from digitization of two polynomials of degree less or equal to $n$, with the same least squares polynomial fit, i.e., with $a_{n}(\gamma)=a_{n}(\beta), a_{n-1}(\gamma)=a_{n-1}(\beta)$, $\ldots, a_{0}(\gamma)=a_{0}(\beta)$. The negative answer will be given. This means that digital polynomial segments and their least squares polynomial fits are in one-to-one correspondence.

Theorem 2 Let $P_{m}\left(\gamma, x_{1}\right)$ and $P_{m}\left(\beta, x_{1}\right)$ be two digital polynomial segments. If $a_{n}(\gamma), a_{n-1}(\gamma), \ldots$, $a_{0}(\gamma)$ and $a_{n}(\beta), a_{n-1}(\beta), \ldots, a_{0}(\beta)$ are the coefficients of the least squares polynomial fits associated to $P_{m}\left(\gamma, x_{1}\right)$ and $P_{m}\left(\beta, x_{1}\right)$, respectively, then:

$$
\left(a_{n}(\gamma)=a_{n}(\beta) \wedge a_{n-1}(\gamma)=a_{n-1}(\beta) \wedge \ldots \wedge a_{0}(\gamma)=a_{0}(\beta)\right) \Longleftrightarrow P_{m}\left(\gamma, x_{1}\right)=P_{m}\left(\beta, x_{1}\right) .
$$

Proof. The direction

$$
P_{m}\left(\gamma, x_{1}\right)=P_{m}\left(\beta, x_{1}\right) \Rightarrow\left(a_{n}(\gamma)=a_{n}(\beta) \wedge a_{n-1}(\gamma)=a_{n-1}(\beta) \wedge \ldots \wedge a_{0}(\gamma)=a_{0}(\beta)\right)
$$

follows from the definitions. The opposite direction will be proved by a contradiction. 
Let the scatter diagrams of digital polynomial segments $P_{m}\left(\gamma, x_{1}\right)$ and $P_{m}\left(\beta, x_{1}\right)$ be:

$$
P_{m}\left(\gamma, x_{1}\right)=\left\{\left(x_{i}, y_{i}\right), i=1, \ldots, m\right\} \text { and } P_{m}\left(\beta, x_{1}\right)=\left\{\left(x_{i}, y_{i}^{\prime}\right), i=1, \ldots, m\right\}
$$

We start by an interpretation of the sums

$$
Y(\gamma)=\sum_{i=1}^{m} y_{i}, \quad Y X_{1}(\gamma)=\sum_{i=1}^{m} y_{i} x_{i}, \quad Y X_{2}(\gamma)=\sum_{i=1}^{m} y_{i} x_{i}^{2}, \quad \ldots, \quad Y X_{n}(\gamma)=\sum_{i=1}^{m} y_{i} x_{i}^{n},
$$

which appear on the right-hand sides of the equalities of the system (1). For convenience and without loss of generality, in the rest of the proof, we can assume that $y_{i} \geq 0$ and $y_{i}^{\prime} \geq 0, i=1,2, \ldots, m$ (otherwise the translation in the vertical direction by $-\min _{i=1,2, \ldots, \pi n}\left\{y_{i}, y_{i}^{\prime}\right\}$ can be applied).

- $\sum_{i=1}^{m} y_{i}-$ is the number of digital points below the digitized polynomial segment and above the
$x$-axis. - $\sum_{i=1}^{m} x_{i} y_{i}-$ can be understood as the sum of the abscissa values of all digital points lying between the digitized polynomial segment and the $x$-axis.

- $\sum_{i=1}^{m} x_{i}^{2} y_{i}-$ is the sum of the squares of the abscissa values of all digital points lying between the digitized polynomial segment and the $x$-axis.

$-\sum_{i=1}^{m} x_{i}^{n} y_{i}-$ is the sum of $n$-th powers of the abscissa values
digitized polynomial segment and the $x$-axis.

Let us assume, on the contrary, that there exist two different digital polynomial segments $P_{m}\left(\gamma, x_{1}\right)$ and $P_{m}\left(\beta, x_{1}\right)$, where the degrees of polynomials $\gamma$ and $\beta$ are at most $n$, with the same associated coefficients of the least squares polynomial fits, i.e., with $a_{n}(\gamma)=a_{n}(\beta), a_{n-1}(\gamma)=a_{n-1}(\beta), \ldots$, $a_{0}(\gamma)=a_{0}(\beta)$. Also, let the digital polynomial segments $P_{m}\left(\gamma, x_{1}\right)$ and $P_{m}\left(\beta, x_{1}\right)$ be obtained by digitization of the polynomial segments $\gamma$ and $\beta$, respectively. From the uniqueness of the solution of the system (1), it follows that there must be

$$
\begin{aligned}
Y(\gamma) & =\sum_{i=1}^{m} y_{i}=\sum_{i=1}^{m} y_{i}^{\prime}=Y(\beta) ; \\
Y X_{1}(\gamma) & =\sum_{i=1}^{m} x_{i} y_{i}=\sum_{i=1}^{m} x_{i} y_{i}^{\prime}=Y X_{1}(\beta) ; \\
Y X_{2}(\gamma) & =\sum_{i=1}^{m} x_{i}^{2} y_{i}=\sum_{i=1}^{m} x_{i}^{2} y_{i}^{\prime}=Y X_{2}(\beta) ; \\
Y X_{n}(\gamma) & =\sum_{i=1}^{m} x_{i}^{n} y_{i}=\sum_{i=1}^{m} x_{i}^{n} y_{i}^{\prime}=Y X_{n}(\beta) .
\end{aligned}
$$

Let us denote the set of digital points belonging to the area $A$, below the polynomial $\gamma$ and above $\tau$-axis, and let us denote the set of digital points belonging to the area $B$, below the polynomial $\beta$ and above the $x$-axis. Let for a digital point $\Delta, x(\Delta)$ and $y(\Delta)$ denote the abscissa and ordinate of $\Delta$, respectively; in other words, $\Delta=(x(\Delta), y(\Delta))$. Then the equalities of (3) and the above interpretation of the sums of (3) give:

$$
\begin{aligned}
\sum_{\Delta \in A \backslash B} 1 & =\sum_{\Delta \in B \backslash A} 1 \\
\sum_{\Delta \in A \backslash B} x(\Delta) & =\sum_{\Delta \in B \backslash A} x(\Delta)
\end{aligned}
$$




$$
\begin{aligned}
\sum_{\Delta \in A \backslash B} x(\Delta)^{2} & =\sum_{\Delta \in B \backslash A} x(\Delta)^{2} \\
\sum_{\Delta \in A \backslash B} x(\Delta)^{n} & =\sum_{\Delta \in B \backslash A} x(\Delta)^{n} .
\end{aligned}
$$

Let $y_{\gamma}(x)=a_{n} \cdot x^{n}+a_{n-1} \cdot x^{n-1}+\ldots+a_{1} \cdot x+a_{0}$ be the equation of the polynomial $\gamma$. An important remark is that $y_{\gamma}(x(\Delta))-y(\Delta)>0$ for $\Delta \in A \backslash B$ while $y_{\gamma}(x(\Delta))-y(\Delta)<0$ for $\Delta \in B \backslash A$.

$$
\begin{aligned}
& \text { If } \sum_{\Delta \in A \backslash B} y(\Delta) \geq \sum_{\Delta \in B \backslash A} y(\Delta) \text {, then a contradiction can be made in the following way: } \\
& 0<\sum_{\Delta \in A \backslash B}\left(\left(a_{n} \cdot x(\Delta)^{n}+a_{n-1} \cdot x(\Delta)^{n-1} \ldots+a_{1} \cdot x(\Delta)+a_{0} \cdot 1\right)-y(\Delta)\right)= \\
& =a_{n} \cdot \sum_{\Delta \in A \backslash B} x(\Delta)^{n}+a_{n-1} \cdot \sum_{\Delta \in A \backslash B} x(\Delta)^{n-1}+\ldots+a_{1} \cdot \sum_{\Delta \in A \backslash B} x(\Delta)+a_{0} \cdot \sum_{\Delta \in A \backslash B} 1-\sum_{\Delta \in A \backslash B} y(\Delta) \leq \\
& \leq a_{n} \cdot \sum_{\Delta \in B \backslash A} x(\Delta)^{n}+a_{n-1} \cdot \sum_{\Delta \in B \backslash A} x(\Delta)^{n-1}+\ldots+a_{1} \cdot \sum_{\Delta \in B \backslash A} x(\Delta)+a_{0} \cdot \sum_{\Delta \in B \backslash A} 1-\sum_{\Delta \in B \backslash A} y(\Delta)= \\
& \left.=\sum_{\Delta \in B \backslash A}\left(a_{n} \cdot x(\Delta)^{n}+a_{n-1} \cdot x(\Delta)^{n-1}+\ldots+a_{1} \cdot x(\Delta)+a_{0} \cdot 1\right)-y(\Delta)\right)<0 .
\end{aligned}
$$

The first and the last inequality are strict because the set differences $A \backslash B$ and $B \backslash A$ are nonempty (the difference of the considered digital polynomial segments is assumed).

$\beta$, instead of $\gamma$.

If $\sum_{\Delta \in A \backslash B} y(\Delta)<\sum_{\Delta \in B \backslash A} y(\Delta)$, then an analogous contradiction can be made with the polynomial

\section{Comments and conclusion}

The least squares fitting technique is applied to digital polynomial segments. It is shown that digital polynomial segments and their least squares polynomial fits are in one-to-one correspondence provided that the degree of digitized polynomials is bounded. This result enables us to define an efficient representation for digital polynomial segments that results from digitization of polynomials with bounded degree, say $n$. Such a representation requires only $n+3$ parameters. Two of them are integers, while the rest of them are rationals.

\section{References}

[1] Aitken, A. C. (1956). Determinants and Matrices, Oliver and Boyd.

[2] Arcelli, L. and Masarotti, A. (1978). On the parallel generation of straight digital lines, Computer Graphics and Inage Processing, 7, 67-83.

[3] Bongiovanni, G, Luccio, G. F. and Zurat A. (1975). The discrete equation of a straight line, IEEE Trans. Comput., 24, 310-313.

[4] Boshernitzan, M. and Fraenkel, A. S. (1981). Nonhomogeneous spectra of numbers, Discrete Mathematics, 34, 325-327.

[5] Boshernitzan, M. and Fraenkel, A. S. (1984). A linear algorithm for nonhomogeneous spectra of numbers, J. Algorithms, 5, 187-198.

[6] Burr, I. W. (1974). Applied Statistical Methods, Academic Press. 
[7] Dorst, L. and Smeulders, A. W. M. (1984). Discrete representation of straight lines, IEEE Trans. Pattern Analysis and Machine Intelligence, 6, 450-463.

[8] Graham, R. L., Lin. and Lin, C.-S. (1978). Spectra of numbers, Mathematics Magazine, 51, 174-176.

[9] Kim, C. E. (1984). Digital disks, IEEE Trans. Pattern Analysis and Machine Intelligence, 6, 372-374.

[10] Klassman, H. (1975). Some aspects of the accuracy of the approximated position of a straight line on a square grid, Computer Graphics and Image Processing, 4, 225-235.

[11] Klette, R., Stojmenović, I. and Zunić J. (1996). A parametrization of digital planes by least square fits and generalizations, Graphical Models and Image Processing, 58, No. 3, 295-300.

[12] Krechmar, V. A. (1974). A Problem Book in Algebra. Mir Publishers, Moscow.

[13] Kovalevsky, V. A. (1990). New definition and fast recognition of digital straight segments and arcs, IEEE Proc. of Tenth Int. Conf. on Pattern Recognition, 10662, 31-34.

[14] Lindenbaum, M. and Koplowitz, J. (1991). A new parametrization of digital straight lines, IEEE Trans. Pattern Analysis and Machine Intelligence, 13, 847-852.

[15] Melter, R. A. and Rosenfeld, A. New views of linearity and connectedness in digital geometry, Pattern Recognition Letters, 10, 9-16.

[16] Melter, R. A., Stojmenović, I. and Žunić, J. (1993). A new characterization of digital lines by least square fits, Pattern Recognition Letters, 14, 83-88.

[17] Nakamura, A. and Aizawa, K. (1984). Digital circles, Computer Vision, Graphics and Image Processing, 26, 242-255.

[18] Rosenfeld, A. (1974). Digital straight line segments, IEEE Trans. Comput., 23, 1264-1269.

[19] Sauer, P. (1993). On the recognition of digital circles in linear time, Computational Geometry: Theory and Applications, 2, 287-302.

[20] Žunić, J. (1995). A coding scheme for certain sets of digital curves, Pattern Recognition Letters, 16, 97-104.

[21] Žunić, J. and Koplowitz, J. (1994). A representation of digital parabolas by least square fits, SPIE Proc,, 2356, 71-78. 